\title{
The Design of Control Strategies Tolerant to Unidentified Failures in Kinematically Redundant Manipulators
}

\author{
M. Goel, A. A. Maciejewski, and V. Balakrishnan \\ Purdue University \\ School of Electrical and Computer Engineering \\ 1285 Electrical Engineering Building \\ West Lafayette, Indiana 47907-1285
}

\begin{abstract}
The use of robots in hostile environments significantly increases the likelihood of failures in the robot's subsystems. Existing techniques for developing failure tolerant robots rely on effective failure detection and identification. Since failure identification is itself a difficult process that may not always be successful it is important to consider the behavior of the robot prior to identification of a fault, or even the possibility of failures remaining unidentified. This work proposes control strategies that improve local measures of failure tolerance for kinematically redundant robots experiencing unidentified locked-joint failures. Measures to evaluate the fault tolerance capability of the various schemes are presented and the performance of the proposed schemes are demonstrated with an example.
\end{abstract}

\section{INTRODUCTION}

Robots are being increasingly used to replace humans for applications in hazardous environments. While failures are not uncommon in industrial robots [1], the likelihood of failures is far greater when robots are applied in harsh environments [2]. Since the very nature of these environments does not allow immediate human intervention for repair or recovery, the ability of a robot to cope with the failures becomes desirable. Common methods of making robots failure tolerant incorporate some form of redundancy in the design; either through duplication of components [3] or through kinematic redundancy $[4,5]$. Existing failure-tolerance schemes, however, rely on successful failure detection and identification; only after a failure is identified is an appropriate failure recovery strategy initiated $[6,7]$. Since failure identification is itself a difficult process that may not always be successful [8], it is essential to consider the possibility of delayed failure identification, or even that of failures remaining unidentified, when developing any effective failure-tolerant control technique.

While there are several ways in which a robot may

This work was supported in part by Sandia National Laboratories under contract no. AL-3011, the Office of Naval Research under contract no. N00014-97-1-0640, and NSF under contract no. MIP-9708309. fail, one common failure mode is a "locked joint", where the affected joint's velocity is identically zero. When such a failure remains unidentified by the robot controller, very large and often unpredictable deflections of the robot end effector can result. Such behavior is clearly unacceptable in cluttered environments due to the increased probability of collisions, and also in the case of teleoperated systems because of operator disorientation caused by the unexpected motions.

This work focuses on developing control strategies that improve local measures of failure tolerance for kinematically redundant manipulators experiencing unidentified locked-joint failures. A general class of tasks characterized by sequences of point-to-point moves in task space is considered.

\section{Mathematical Framework}

The position and/or orientation (henceforth referred to as "position") of the end effector of a manipulator can be expressed in terms of its joint variables by the kinematic equation

$$
\mathbf{x}=f(\mathbf{q}),
$$

where $\mathbf{x} \in \mathbb{R}^{m}$ is the position of the end effector, $\mathbf{q} \in \mathbb{R}^{n}$ is the vector of joint variables, and $m$ and $n$ the dimensions of the task space and joint space respectively. Manipulators that have more degrees of freedom (DOFs) than required for a task, i.e., $n>m$, are said to be redundant. The end-effector velocity is expressed in terms of the joint rates as

$$
\dot{\mathbf{x}}=J \dot{\mathrm{q}},
$$

where $J \in \mathbb{R}^{m \times n}$ is the manipulator Jacobian, $\dot{\mathrm{x}}$ is the end-effector velocity, and $\dot{q}$ is the joint velocity.

If perfect servo control of the joints is assumed, then in a healthy manipulator the actual joint velocity $\dot{\mathbf{q}}_{\mathrm{a}}$ equals the commanded velocity $\dot{\mathbf{q}}_{\mathrm{c}}$. However, in the event of a locked-joint failure of the $i$-th joint, the corresponding element of $\dot{\mathbf{q}}_{\mathbf{a}}$ is identically zero. Then, the 
actual end-effector velocity is given by

$$
\dot{\mathbf{x}}_{\mathrm{a}}={ }^{i} J \dot{\mathbf{q}}_{\mathrm{c}}
$$

where ${ }^{i} J$ is the post-failure Jacobian, given by

$$
{ }^{i} J=\left[\begin{array}{lllllll}
\mathbf{j}_{1} & \cdots & \mathbf{j}_{i-1} & \mathbf{0} & \mathbf{j}_{i+1} & \cdots & \mathbf{j}_{n}
\end{array}\right] .
$$

A common method for generating $\dot{q}$ is the inverse kinematic scheme

$$
\dot{\mathbf{q}}=G \dot{\mathbf{x}},
$$

where $G$ is a generalized inverse of $J$ satisfying the Penrose condition $J G J=J$. A frequently encountered generalized inverse is the pseudoinverse $J^{+}$, which yields the least squares minimum norm solution. For full rank $J$, the pseudoinverse can be expressed as $J^{+}=J^{T}\left(J J^{T}\right)^{-1}$.

In this work a general class of tasks characterized by sequences of point-to-point moves is considered. The commanded end-effector velocity is simply straight line motion towards the desired task position $\mathrm{x}_{\mathrm{d}}$ :

$$
\dot{\mathbf{x}}_{\mathrm{c}}=K_{\mathrm{e}}\left(\mathbf{x}_{\mathrm{d}}-\mathbf{x}_{\mathrm{a}}\right),
$$

where $\mathbf{x}_{\mathbf{a}}$ is the actual position of the end effector and $K_{\mathrm{e}}$ is a constant position error gain that is adjusted when necessary to limit the commanded end-effector velocity to a maximum allowable value.

In the event of a locked-joint failure, the actual endeffector velocity in general will not be as commanded by (6). In particular, if joint $i$ fails then the actual end-effector velocity is given by

$$
\dot{\mathbf{x}}_{\mathrm{a}}=\left({ }^{i} J G\right) \dot{\mathbf{x}}_{\mathrm{c}}=J\left({ }^{i} I \dot{\mathbf{q}}_{\mathrm{c}}\right),
$$

where $G$ is a generalized inverse of $J$. Thus $\dot{\mathbf{x}}_{\mathrm{a}}$ will equal $\dot{\mathbf{x}}_{\mathrm{c}}$ only if the failed joint is not commanded to move so that ${ }^{i} I \dot{\mathbf{q}}_{\mathrm{c}}=\dot{\mathbf{q}}_{\mathrm{c}}$.

With a focus on the local effects of failures, the error in the end-effector velocity is of primary concern. This error can be measured in a number of different ways. Two fundamental measures considered here are the expected and the peak velocity errors. These error measures and techniques for their minimization are discussed next.

\section{Minimizing Expected Velocity ERror}

If all joints fail with equal probability, then, for an arbitrary commanded end-effector velocity $\dot{\mathbf{x}}_{\mathrm{c}}$, the expected error $\left(E_{\mathrm{e}}\right)$ in the end-effector velocity due to the failure of an arbitrary joint can be written as

$$
E_{\mathrm{e}}=\frac{1}{n} \sum_{i=1}^{n}\left\|\dot{\mathrm{x}}_{\mathrm{c}}-\dot{\mathrm{x}}_{\mathrm{a}}\right\|^{2} .
$$

For full-rank $J$ a weighted generalized inverse $J^{w}$ is defined as

$$
J^{w}=W^{-1} J^{T}\left(J W^{-1} J^{T}\right)^{-1},
$$

where $W$ is a weighting matrix. Then, $J J^{w}=I$ so that $\dot{\mathbf{x}}_{\mathrm{c}}=J \dot{\mathrm{q}}_{\mathrm{c}}$. From $(7) \dot{\mathbf{x}}_{\mathrm{a}}={ }^{i} J \dot{\mathrm{q}}_{\mathrm{c}}$, so the expected error can then be rewritten as

$$
E_{\mathrm{e}}=\frac{1}{n} \sum_{i=1}^{n}\left\|\mathbf{j}_{i}\right\|^{2} \dot{\mathrm{q}}_{\mathrm{c}}(i)^{2} .
$$

From (10) it is seen that the expected error in the endeffector velocity is equal to a weighted sum of the velocities of the different joints, a formulation that makes it a natural candidate for minimization by a weighted inverse. A diagonal weighting matrix $W$, with the $i$-th diagonal entry assigned a value equal to the norm squared of the associated column of the Jacobian, minimizes the expected error $E_{\mathrm{e}}$. Weighting the joints in this manner seeks to balance the contributions of the joints to the end-effector motion so that the failure of any one joint can result in an error no greater than that resulting from the failure of any other joint.

\section{Minimizing PeAK Velocity Error}

\section{A. Introduction}

While the minimization of the expected velocity error through the use of a weighted inverse offers a simple and reasonably effective method of minimizing the performance degradation due to the local effects of a failure, it is possible to further enhance the post-failure performance by making use of additional available information, and by optimizing a measure other than the expected error. Since information about the commanded end-effector velocity is available to the robot controller for on-line computation of joint rates, incorporating that knowledge in the computation of an optimal inverse is naturally very effective. In addition, since the worst-case error resulting from the failure of a joint is often more critical than the "expected error", it is more meaningful to focus on the peak error. One error measure that addresses both these issues is $\operatorname{PKE}\left(\dot{\mathbf{x}}_{\mathrm{c}}\right)$, defined as

$$
\operatorname{PKE}\left(\dot{\mathbf{x}}_{\mathrm{c}}\right)=\max _{i}\left\|\dot{\mathbf{x}}_{\mathrm{c}}-\left({ }^{i} J J^{\#}\right) \dot{\mathbf{x}}_{\mathrm{c}}\right\|,
$$

where $J^{\#}$ is not necessarily a generalized inverse. It is easily shown that $\mathrm{PKE}\left(\dot{\mathrm{x}}_{\mathrm{c}}\right)$ provides well-defined bounds for other measures such as mean-squared error for a given $\dot{x}_{c}$, and the peak angular deviation between $\dot{\mathbf{x}}_{\mathbf{c}}$ and $\dot{\mathbf{x}}_{\mathbf{a}}$ [11]. Since $\operatorname{PKE}\left(\dot{\mathbf{x}}_{\mathbf{c}}\right)$ in fact represents the "peak error", it also reflects the worst-case possible deviation of the end effector from the desired straight line trajectory toward the target. Thus, guaranteeing an appropriate bound on this measure is one way of also ensuring global convergence. 
For these reasons, minimizing $\operatorname{PKE}\left(\dot{\mathbf{x}}_{\mathrm{c}}\right)$ is the primary focus of the rest of this work. This optimization problem can be written as follows:

Problem: Minimize $\operatorname{PKE}\left(\dot{\mathbf{x}}_{\mathbf{c}}\right)$

Given a manipulator at configuration $q$, with Jacobian $J$, and desired end-effector velocity $\dot{\mathbf{x}}_{\mathrm{c}}$, compute an inverse $J^{\#}$ such that

- The post-failure error, as measured by $\operatorname{PKE}\left(\dot{\mathbf{x}}_{\mathrm{c}}\right)$, is minimized

- The pre-failure error is bounded by $\epsilon_{\mathrm{pre}}$, the maximum allowable pre-failure error

The problem is formally stated as

Min. $\max _{i}\left\|\dot{\mathbf{x}}_{\mathrm{c}}-\left({ }^{i} J J^{\#}\right) \dot{\mathbf{x}}_{\mathrm{c}}\right\| \quad i=1, \ldots, n, \quad$ over $J^{\#}$ S.t. $\left\|\dot{\mathbf{x}}_{\mathrm{c}}-\left(J J^{\#}\right) \dot{\mathbf{x}}_{\mathrm{c}}\right\|<\epsilon_{\mathrm{pre}}$

The inequality condition in (12) implies that the prefailure performance of the manipulator may be intentionally compromised in solving for the optimal $J^{\#}$, thereby offering the potential of improving the postfailure performance. Problem (12) can be reduced to the special case requiring uncompromised pre-failure behavior simply by setting $\epsilon_{\text {pre }}$ to zero.

\section{B. An LMI-Based Inverse: A Globally Optimal Ap- proach to Minimizing Peak Velocity Error}

Unlike the minimization of the expected error where a closed-form solution could be derived, an analytical solution to Problem (12) is not easily defined; it must thus be numerically solved. Linear matrix inequality (LMI) based convex optimization techniques can be used to obtain a globally optimal solution to the problem. Using simple mathematical manipulation, Problem (12) can be rewritten as

$$
\text { Sin. } \quad\left[\begin{array}{cc}
\gamma \text { over } J^{\#} & \\
\gamma I & \dot{\mathrm{x}}_{\mathrm{c}}^{T}\left(I-{ }^{i} J J^{\#}\right)^{T} \\
\left(I-{ }^{i} J J^{\#}\right) \dot{\mathrm{x}}_{\mathrm{c}} & \gamma I \\
\epsilon_{\mathrm{pre}} I & \dot{\mathrm{x}}_{\mathrm{c}}^{T}\left(I-J J^{\#}\right)^{T} \\
\left(I-J J^{\#}\right) \dot{\mathbf{x}}_{\mathrm{c}} & \epsilon_{\mathrm{pre}} I
\end{array}\right]>0
$$

The inequalities in (13) are linear matrix inequalities, with each inequality denoting the required positivedefiniteness of the matrix on the left-hand side.

It turns out that the optimization problem (13) falls into a general class of LMI-based convex optimization problems. There exist very effective and powerful algorithms for numerically computing solutions to such problems that achieve a global optimum in polynomial time, with non-heuristic stopping criteria [12]. Moreover, software packages for solving LMI-based optimization problems are readily available [12]. With the desired optimization problem formulated in the LMI framework as in (13), any of the available software tools can be used to solve for the optimal failure-tolerant inverse as defined here.

\section{A Damped Least-Squares Based Inverse: An Alter- native Approach to Minimizing Peak Velocity Error}

While the LMI-based method yields a globally optimal solution to Problem (12), the time required to compute a solution with currently available tools may make it undesirable for some applications. One approach to reducing the amount of computation required is to settle for a close-to-optimal solution that can be computed much faster. This may be done by restricting the structure of $J^{\#}$, as done here by using a damped leastsquares inverse [13-15]. In particular, we rewrite (12) in terms of the damped least-squares inverse $J^{\lambda}$ as

$$
\begin{aligned}
& \text { Min. } \max _{i}\left\|\dot{\mathbf{x}}_{\mathrm{c}}-\left({ }^{i} J J^{\lambda}\right) \dot{\mathbf{x}}_{\mathrm{c}}\right\| \quad i=1, \ldots, n, \quad \text { over } \lambda \\
& \text { S.t. }\left\|\dot{\mathbf{x}}_{\mathrm{c}}-\left(J J^{\lambda}\right) \dot{\mathbf{x}}_{\mathrm{c}}\right\|<\epsilon_{\mathrm{pre}},
\end{aligned}
$$

where $J^{\lambda}=J^{T}\left(J J^{T}+\lambda^{2} I\right)^{-1}$ (assuming $\lambda^{2} \neq 0$ when $J$ is rank deficient).

The inequality constraint of (14) simply represents a bound on the end-effector velocity error of the healthy manipulator. Since this error is in fact a monotonic function of the damping factor [16], this constraint defines an upper bound, $\lambda_{\mathrm{U}}$, on the damping factor $\lambda$.

Problem (14) requires the minimization of a maximum of $n$ functions, i.e., a minimax problem. While its solution using classical descent techniques is difficult, a numerical technique is proposed here that is guaranteed to find the optimal solution to within a pre-specified tolerance. This technique is inspired by the bisection method presented in [17].

Let ${ }^{i} \mathbf{e}_{\lambda}$ define the error in the end-effector velocity due to the failure of joint $i$ when using the damped least-squares inverse $J^{\lambda}$, i.e.,

$$
{ }^{i} \mathbf{e}_{\lambda}=\dot{\mathbf{x}}_{c}-\left({ }^{i} J J^{\lambda}\right) \dot{\mathbf{x}}_{\mathrm{c}} .
$$

Then, for $\gamma>0, \gamma \neq\left\|\dot{x}_{\mathrm{c}}\right\|$, and a matrix ${ }^{i} M_{\gamma}$ defined as

$$
\begin{aligned}
& { }^{i} M_{\gamma}= \\
& \quad\left[\begin{array}{cc}
\dot{\mathbf{x}}_{\mathrm{c}} \dot{\mathrm{x}}_{\mathrm{c}}^{T} R^{-1}\left({ }^{i} J J^{T}\right)-J J^{T} & \frac{1}{\gamma} \dot{\mathrm{x}}_{\mathrm{c}}\left(\dot{\mathrm{x}}_{\mathrm{c}}^{T} R^{-1} \dot{\mathrm{x}}_{\mathrm{c}}-1\right) \dot{\mathrm{x}}_{\mathrm{c}}^{T} \\
\gamma\left({ }^{i} J J^{T}\right)^{T} R^{-1}\left({ }^{i} J J^{T}\right) & \left({ }^{i} J J^{T}\right)^{T} R^{-1} \dot{\mathbf{x}}_{c} \dot{\mathbf{x}}_{\mathrm{c}}^{T}-J J^{T}
\end{array}\right],
\end{aligned}
$$

where $R=\left(\dot{\mathrm{x}}_{\mathrm{c}} \dot{\mathbf{x}}_{\mathrm{c}}^{T}-\gamma^{2} I\right)$, we have,

Theorem 1: (a) Let $\gamma<\left\|^{i} \mathbf{e}_{\lambda}\right\|$ for some $\lambda^{2}$. Then $\left\|{ }^{i} \mathbf{e}_{\lambda}\right\|_{\min } \leq \gamma$ if and only if ${ }^{i} M_{\gamma}$ has positive real eigenvalues (i.e., at least one). (b) The positive real eigenvalues of $M_{\| i} \mathbf{e}_{\lambda} \|_{\min }$ are exactly the values of $\lambda^{2}$ for which $\left\|\mathbf{e}_{\lambda}\right\|=\left\|{ }^{i} \mathbf{e}_{\lambda}\right\|_{\min }$.

Theorem 1 implies that if an error estimate $\gamma$ is known to lie below the peak of an error function, i.e., 
$\left\|{ }^{i} \mathbf{e}_{\lambda}\right\|$, then based on the eigenvalues of the corresponding ${ }^{i} M_{\gamma}$ it can be determined if the estimate lies above or below the minimum of the function. This idea is combined with a standard bisection procedure to determine the minimum of a single, or a set of error functions. The failure of an arbitrary joint can be considered by simply considering the worst-case error defined as

$$
\left\|^{\mathrm{wc}} \mathbf{e}_{\lambda}\right\|_{\min }=\min _{\lambda^{2} \in \mathbb{R}}\left(\max _{i}\left\|^{i} \mathbf{e}_{\lambda}\right\|\right)
$$

The details of the algorithm are presented in [11].

\section{MEasures of Performance}

The ten measures of performance used in this study are summarized in Table V. While some of these measures have been incorporated from previous work on performance evaluation of teleoperated systems [18-22], additional measures focusing specifically on failure related performance issues are also proposed. One way of graphically representing all of these measures at the same time is illustrated in Fig. 2, where each measure, normalized by its maximum value over all the different cases (tasks/control schemes) being compared, is represented as a shaded fraction of a 1/10th-area sector of a circle of unit radius. Based on this representation, a cumulative performance measure (CPM) is defined as

$$
\mathrm{CPM}=\sum_{i=1}^{10} \frac{\mathrm{M}_{i}}{\max \left(\mathrm{M}_{i}\right)}\left(\frac{\pi}{10}\right) .
$$

The CPM denotes the total shaded-area of the unit circle.

\section{A 5-DOF SPatial Positioning Example}

The control strategies developed in the previous sections were compared using the performance measures defined in Section V. A Puma 550 robot with a $0.56 \mathrm{~m}$ wrist offset was used to perform spatial positioning tasks, resulting in two degrees of redundancy (see Fig. 3).

To study "typical" behavior encountered with failures, a set of randomly generated cases was considered. Specifically, one thousand point-to-point motion tasks were generated, each specified by the initial configuration and final end-effector position of the robot. The failure of joint 2 was assumed in each of these cases and the desired task positions were chosen to be reachable after the failure. The distance between the initial and desired task-positions was in the range $1.0-1.1 \mathrm{~m}$. In each of these tasks the robot was commanded to move directly toward the target with a maximum allowable end-effector velocity of $\left\|\dot{x}_{c}\right\|_{\max }=0.05 \mathrm{~m} / \mathrm{cyc}$ until the task was completed. (The joint-rate norm was
TABLE I

\begin{tabular}{|c|c|}
\hline Measure & Description \\
\hline $\mathrm{M}_{1}$ & $\begin{array}{l}\text { Task Completion Time (cycles) }\left(\mathrm{TCT}_{\text {cyc }}\right) \text { : Com- } \\
\text { puted in terms of the number of controller cy- } \\
\text { cles rather than in elapsed time. Allows control } \\
\text { schemes to be evaluated independent of the un- } \\
\text { derlying computational cost. }\end{array}$ \\
\hline $\mathrm{M}_{2}$ & $\begin{array}{l}\text { Task Completion Time (seconds })\left(\mathrm{TCT}_{\mathrm{secs}}\right): \mathrm{A} \\
\text { measure of the elapsed time. }\end{array}$ \\
\hline$\overline{M_{3}}$ & $\begin{array}{l}\text { Total Path Length (TPL): The total length of the } \\
\text { end-effector trajectory. }\end{array}$ \\
\hline$\overline{\mathrm{M}_{4}}$ & $\begin{array}{l}\text { Peak Trajectory Deviation (Magnitude) } \\
\text { (PTD mag): The maximum displacement of the } \\
\text { end effector from the desired straight-line trajec- } \\
\text { tory, measured over the entire task. }\end{array}$ \\
\hline $\mathrm{M}_{5}$ & $\begin{array}{l}\left.\text { Peak Trajectory Deviation (Angle) ( } \mathrm{PTD}_{\text {ang }}\right) \text { : } \\
\text { The maximum angular deviation of the end ef- } \\
\text { fector from the desired straight-line trajectory, } \\
\text { measured from the initial end-effector position, } \\
\text { over the entire task. }\end{array}$ \\
\hline $\mathrm{M}_{6}$ & $\begin{array}{l}\text { Peak End-Effector Velocity Error (Magnitude) } \\
\text { (PVE } \text { Pag }_{\text {mag }} \text { The peak magnitude of the difference } \\
\text { in the commanded end-effector velocity } \dot{\mathbf{x}}_{\mathrm{c}} \text { and } \\
\text { its actual value } \dot{\mathbf{x}}_{\mathrm{a}} \text {, measured over the the entire } \\
\text { task. }\end{array}$ \\
\hline $\mathrm{M}_{7}$ & $\begin{array}{l}\text { Mean End-Effector Velocity Error (Magnitude) } \\
\text { (MVE } \text { mag }_{\text {mag }} \text { ) The mean magnitude of the differ- } \\
\text { ence in the commanded end-effector velocity } \dot{\mathrm{x}}_{\mathbf{c}} \\
\text { and its actual value } \dot{\mathbf{x}}_{\mathbf{a}} \text {, computed over the entire } \\
\text { task. }\end{array}$ \\
\hline $\mathrm{M}_{8}$ & $\begin{array}{l}\text { Peak End-Effector Velocity Error (Angle) } \\
\left.\text { (PVE }{ }_{\text {ang }}\right) \text { : The peak angular deviation between } \\
\text { the commanded end-effector velocity } \dot{\mathrm{x}}_{\mathrm{c}} \text { and its } \\
\text { actual value } \dot{\mathrm{x}}_{\mathrm{a}} \text {, measured over the the entire } \\
\text { task. }\end{array}$ \\
\hline $\mathrm{M}_{9}$ & $\begin{array}{l}\text { Mean End-Effector Velocity Error (Angle) } \\
\left.\text { (MVE }{ }_{\text {ang }}\right) \text { : The mean angular deviation between } \\
\text { the commanded end-effector velocity } \dot{\mathrm{x}}_{\mathrm{c}} \text { and its } \\
\text { actual value } \dot{\mathrm{x}}_{\mathrm{a}} \text {, computed over the entire task. }\end{array}$ \\
\hline $\mathrm{M}_{10}$ & $\begin{array}{l}\text { Number of Trajectory Corrections (NTC): The } \\
\text { total number of corrections made by the opera- } \\
\text { tor/computer to compensate for any increase in } \\
\text { the position error of the end-effector. }\end{array}$ \\
\hline
\end{tabular}

Performance measures.

also bounded by $\left\|\dot{\mathbf{q}}_{\mathrm{c}}\right\|_{\max }=0.2 \mathrm{rad} / \mathrm{cyc}$.) The tasks were performed with and without failures, for each of the following five control schemes discussed earlier:

1. Pseudoinverse

2. Weighted inverse

3. LMI-based inverse with no allowable pre-failure error, i.e., $\epsilon_{\text {pre }}=0$

4. LMI-based inverse with $\epsilon_{\text {pre }}=0.5$ for a unit-norm $\dot{\mathbf{x}}_{\mathrm{c}}$ and

5. Damped least-squares inverse, also with $\epsilon_{\text {pre }}=0.5$ for a unit-norm $\dot{x}_{c}$.

Plotted in Fig. 1 are the $\mathrm{PVE}_{\mathrm{mag}}$ values for each of the four failure-tolerant schemes (shown in gray) along with the $\mathrm{PVE}_{\mathrm{mag}}$ of the pseudoinverse (shown using solid black curves), for failure conditions of all thousand 

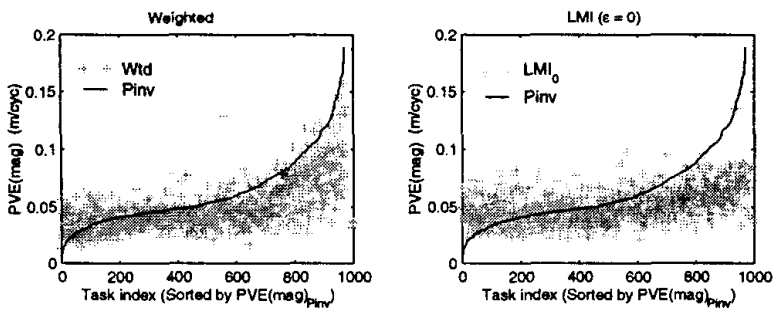

LMI $(\varepsilon=0.5)$
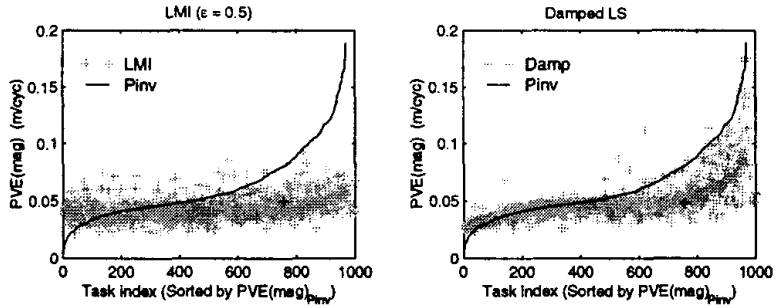

Fig. 1. The $\mathrm{PVE}_{\mathrm{mag}}$ values of the various control schemes plotted for the failure conditions of the one thousand randomly generated tasks. The values for each of the four failure-tolerant schemes are shown using gray plus signs, and those for the pseudoinverse are shown using solid black curves. The task used in the illustrative example is indicated with a black plus sign.

tasks. The task indices plotted on the $x$-axes are sorted based on the PVE $\mathrm{Pag}_{\text {mag }}$ of the pseudoinverse, so that increasing distances along the abscissa indicate increasing levels of performance degradation (in terms of $\mathrm{PVE}_{\mathrm{mag}}$ ) experienced by the pseudoinverse. Based on this representation, a failure-tolerant control scheme performs better than the pseudoinverse for a task if the gray plus sign corresponding to the task lies below the pseudoinverse curve. From the distribution of the gray plus signs about the pseudoinverse curve in these plots it is seen that the proposed schemes clearly outperform the pseudoinverse for tasks with high values of $\mathrm{PVE}_{\mathrm{mag}}$, while being only marginally inferior to the pseudoinverse for tasks with low values of $\mathrm{PVE}_{\mathrm{mag}}$. It is also seen that while the tail end of the pseudoinverse curve rises very sharply, implying rapidly deteriorating performance for the corresponding tasks, the corresponding behavior of the failure tolerant schemes is much better. The gap at the tail of the pseudoinverse curve, representing the range of tasks for which the pseudoinverse was not even able to converge to the desired position, is almost absent for the proposed inverses. In addition, the relatively flat performance curves of the proposed inverses (especially the LMI-based inverses), imply very consistent and predictable behavior. These characteristics of $\mathrm{PVE}_{\mathrm{mag}}$ for the different schemes are representative of those for all measures.

As an illustrative example, one case from the thousand randomly generated cases for which the pseudoinverse demonstrated relatively poor behavior, is discussed next. This special case is characterized by

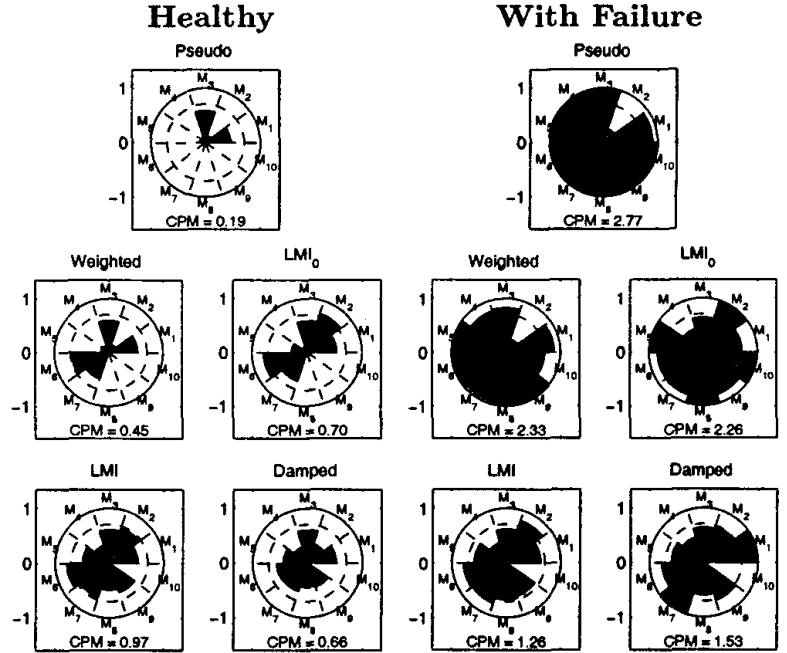

Fig. 2. A graphical representation of the ten performance measures, plotted for the five different control schemes with and without failures. The values of CPM, corresponding to the area of the shaded region, are also shown.

an initial configuration of $\mathrm{q}_{\mathrm{i}}=\left[\begin{array}{llllll}0 & -\pi / 2 & 0 & 0 & 0 & 0\end{array}\right]^{T}$ (i.e., $x_{\mathbf{i}}=\left[\begin{array}{lll}-0.99 & 0.15 & 0.41\end{array}\right]^{T}$ ) and a final position of $\mathbf{x}_{\mathrm{d}}=\left[\begin{array}{lll}-0.30 & 0.50 & 1.10\end{array}\right]^{T}$. The corresponding values of the performance measures, computed for each scheme, are plotted in Fig. 2. This task is highlighted in Fig 1 with black plus signs.

From the performance plots in Fig. 2, it is seen that overall the four proposed failure-tolerant control strategies are able to cope with the failures far better than the pseudoinverse. ${ }^{1}$ The most significant performance improvement in this example is observed for the trajectory deviation measures. The superiority of the LMIbased and the damped least-squares inverses over the weighted inverse are observed for peak-error measures, namely, $\mathrm{PTD}_{\mathrm{mag}}, \mathrm{PTD}_{\mathrm{mag}}, \mathrm{PVE}_{\mathrm{mag}}$ and $\mathrm{PVE}_{\text {ang }}$. In terms of the CPM the LMI-based inverse with $\epsilon_{\text {pre }}=0.5$ performs the best, closely followed by the damped leastsquares inverse.

Figs. 3-5 show the actual trajectories taken by the robot for the following cases: pseudoinverse with failure (Fig. 3); LMI-based inverse with failure (Fig. 4); and LMI-based inverse without failure (Fig. 5). The manipulator exhibits considerable deviation from the commanded straight-line when using the pseudoinverse. The performance of the LMI-based inverse for the same failure condition demonstrates a substantial reduction

${ }^{1}$ The $\mathrm{MVE}_{\mathrm{mag}}$ and $\mathrm{PVE}$ mag are not necessarily zero for a healthy manipulator due to a bound on the physically allowable joint velocity norm $\left\|\dot{\mathbf{q}}_{\mathrm{c}}\right\|$. Since the pseudoinverse produces a minimum norm solution, it suffers the least in terms of the endeffector velocity magnitude errors, resulting from normalization of joint rates, when they exceed $\left\|\dot{q}_{c}\right\|_{\max }$. 

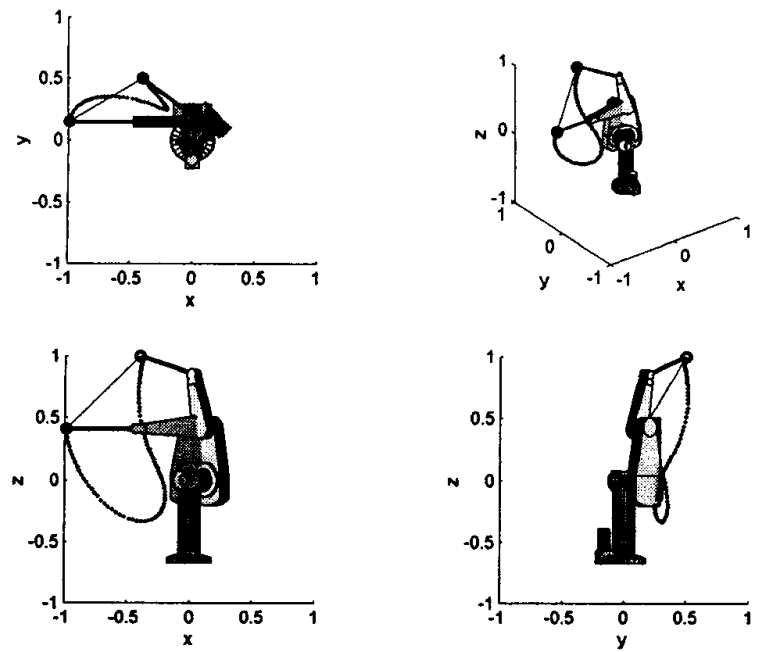

Fig. 3. A Puma 550 with an unidentified failure of Joint 2 performing a spatial point-to-point motion task using pseudoinverse control. The initial configuration and the desired task position are $q_{i}=\left[\begin{array}{lllllll}0 & -\pi / 2 & 0 & 0 & 0 & 0\end{array}\right]^{T}$ (i.e., $x_{i}=\left[\begin{array}{llll}-0.99 & 0.15 & 0.41\end{array}\right]^{T}$ ) and $x_{\mathrm{d}}=\left[\begin{array}{lll}-0.30 & 0.50 & 1.10\end{array}\right]^{T}$ respectively.
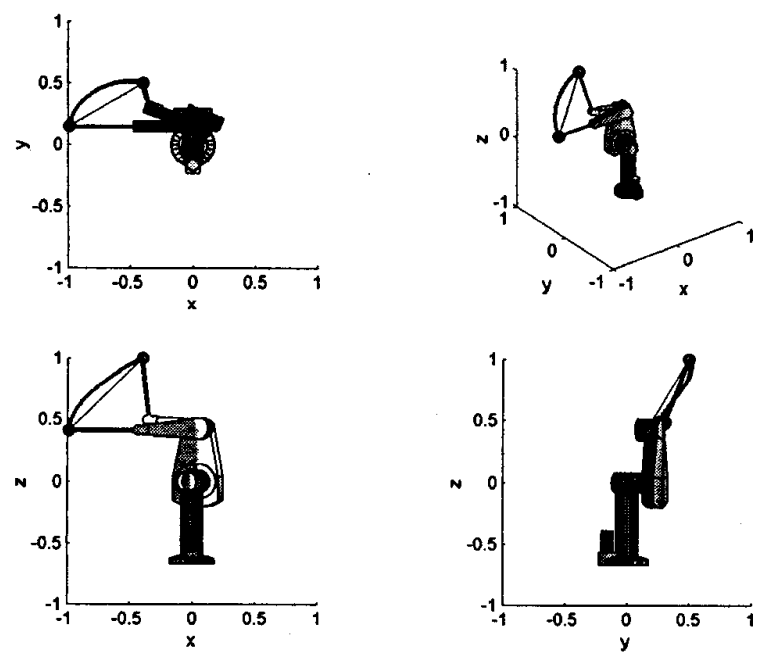

Fig. 4. A Puma 550 with an unidentified failure of Joint 2 performing a spatial point-to-point motion task (as in Fig. 3) using the LMI-based control inverse with $\epsilon_{\text {pre }}=0.5$.

in the overall deviation from the desired trajectory, while producing only a slight degradation in the performance of the healthy manipulator.

\section{CONCLUSIONS}

Three different failure-tolerant inverse kinematic schemes were proposed for dealing with unidentified locked-joint failures. All of them resulted in post-
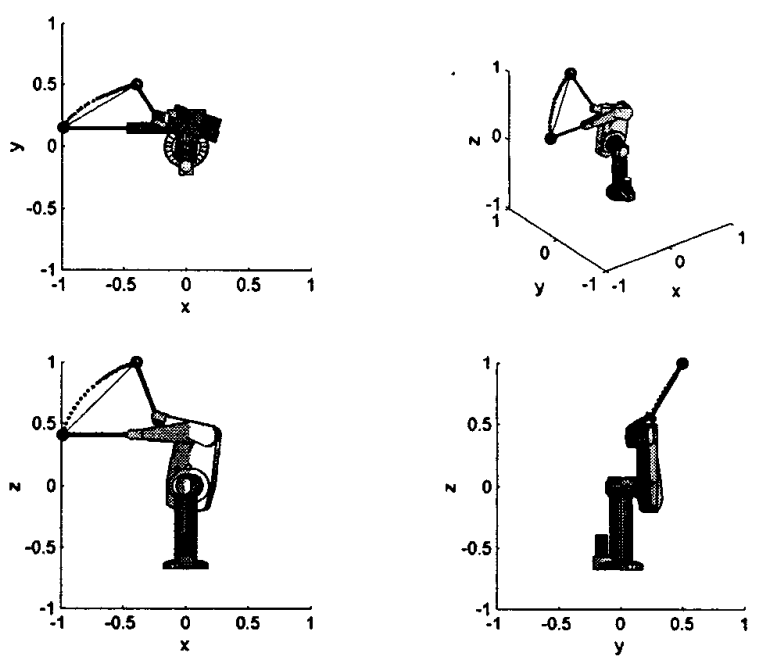

Fig. 5. A healthy Puma 550 performing a spatial point-to-point motion task (as in Fig. 3) using the LMI-based control inverse with $\epsilon_{\text {pre }}=0.5$.

failure performance that was superior to that of the pseudoinverse. While the globally optimal LMI-based inverse resulted in the best post-failure performance, especially for worst-case scenarios, the damped leastsquares based inverse represented a good tradeoff between approximating optimal behavior with very little computational expense. These inverses can also be used to complement failure detection and identification schemes in order to mitigate the effects of failures prior to them being identified and appropriately handled.

\section{REFERENCES}

[1] B. S. Dhillon, Robot Reliability and Safety, Springer-Verlag, New York, 1991.

[2] R. Colbaugh and M. Jamshidi, "Robot manipulator control for hazardous waste-handling applications," J. Robot. Syst., vol. 9, no. 2, pp. 215-250, 1992.

[3] E. C. Wu, J. C. Hwang, and J. T. Chladek, "Fault-tolerant joint development for the space shuttle remote manipulator system: Analysis and experiment," IEEE Trans. Robot. Automat., vol. 9, no. 5, pp. 675-684, Oct. 1993.

[4] C. L. Lewis and A. A. Maciejewski, "Dexterity optimization of kinematically redundant manipulators in the presence of failures," Comput. Elect. Eng., vol. 20, no. 3, pp. 273-288, May 1994.

[5] C. J. J. Paredis, W. K. F. Au, and P. K. Khosla, "Kinematic design of fault tolerant manipulators," Comput. Elect. Eng., vol. 20, no. 3, pp. 211-220, May 1994.

[6] M. L. Visinsky, J. R. Cavallaro, and I. D. Walker, "A dynamic fault tolerance framework for remote robots," IEEE Trans. Robot. Automat., vol. 11, no. 4, pp. 477-490, Aug. 1995.

[7] Y. Ting, S. Tosunoglu, and D. Tesar, "A control structure for fault-tolerant operation of robotic manipulators," in Proc. 1993 Int. Conf. Robot. Automat., pp. 684-690, (Atlanta, Georgia), May 2-6 1993. 
[8] M. L. Visinsky, J. R. Cavallaro, and I. D. Walker, "Robotic fault detection and fault tolerance: A survey," Reliab. Eng. Syst. Safety, vol. 46, pp. 139-158, 1994.

[9] M. Goel, A. A. Maciejewski, and V. Balakrishnan, "An analysis of the post-fault behavior of robotic manipulators," in Proc. 1997 Int. Conf. Robot. Automat., pp. 2583-2588, (Albuquerque, NM), Apr. 20-25 1997.

[10] M. Goel, A. A. Maciejewski, and V. Balakrishnan, "Undetected locked-joint failures in kinematically redundant manipulators: A workspace analysis," in Proc. 1998 Int. Conf. Intelligent Robots Syst., pp. 317-322, (Victoria, B.C., Canada), Oct. 12-16 1998.

[11] M. Goel, Tolerating Undetected Failures in Robotic Manipulators, PhD thesis, Purdue University, 1998.

[12] S. Boyd, L. El Ghaoui, E. Feron, and V. Balakrishnan, Linear Matrix Inequalities in System and Control Theory, vol. 15 of Stud. App. Math., SIAM, Philadelphia, PA, Jun. 1994.

[13] C. W. Wampler II, "Manipulator inverse kinematic solutions based on vector formulations and damped least-squares methods," IEEE Trans. Syst., Man, Cyber,, vol. SMC-16, pp. 93-101, Jan./Feb. 1986.

[14] Y. Nakamura and H. Hanafusa, "Inverse kinematic solutions with singularity robustness for robot manipulator control," ASME J. Dyn. Syst., Meas., Control, vol. 108, no. 3, pp. 163-171, Sep. 1986.

[15] M. Ramabhadran. Fault tolerant robots. EE696 Project Report, Purdue University, 1995.
[16] A. A. Maciejewski and C. A. Klein, "Numerical filtering for the operation of robotic manipulators through kinematically singular configurations," $J$. Robot. Syst., vol. 5, no. 6, pp. 527-552, Dec. 1988.

[17] S. Boyd, V. Balakrishnan, and P. Kabamba, "A bisection method for computing the $\mathbf{H}_{\infty}$ norm of a transfer matrix and related problems," Math. Control, Signals, Syst., vol. 2, no. 3 , pp. 207-219, 1989.

[18] V. Lumelsky, "On human performance in telerobotics," IEEE Trans. Syst., Man, Cyber., vol. 21, no. 5, pp. 971982, Sep./Oct. 1991.

[19] G. F. McLean, B. Prescott, and R. Podhorodeski, "Teleoperated system performance evaluation," IEEE Trans. Syst., Man, Cyber., vol. 24, no. 5, pp. 796-804, May 1994.

[20] G. Magenes, J. L. Vercher, and G. M. Gauthier, "Hand movement strategies in telecontrolled motion along 2-D trajectories," IEEE Thans. Syst., Man, Cyber., vol. 22, no. 2, pp. 242-257, Mar./Apr. 1992.

[21] J. V. Draper, S. J. Handel, and C. C. Hood, "The impact of partial joint failure on teleoperation task performance," in M. Jamshidi and P. J. Eicker, Proc. of the 4th ANS Top. Meet. Robot. Rem. Syst., pp. 433-439. Albuquerque, New Mexico, Feb. 24-28, 1991.

[22] D.-Y. Hwang and B. Hannaford, "Teleoperation performance with a kinematically redundant slave robot," Int. J. Robot. Res., vol. 17, no. 6, pp. 579-597, Jun. 1998. 